PURD-TH-96-06, OSU-TA-20/96, hep-ph/9608458

\title{
The Universe after inflation: the wide resonance case
}

\author{
S. Yu. Khlebnikov ${ }^{1}$ and I. I. Tkachev ${ }^{2,3}$ \\ ${ }^{1}$ Department of Physics, Purdue University, West Lafayette, IN 47907 \\ ${ }^{2}$ Department of Physics, The Ohio State University, Columbus, OH 43210 \\ ${ }^{3}$ Institute for Nuclear Research of the Academy of Sciences of Russia \\ Moscow 117312, Russia
}

(August 27, 1996)

\begin{abstract}
We study numerically the decay of massive and massless inflatons into massive excitations, via a $\phi^{2} X^{2}$ coupling, in the expanding Universe. We find that a wide enough resonance can survive the Universe expansion, though account for the expansion is very important for determining precisely how wide it should be. For a massive inflaton, the effective production of particles with mass ten times that of the inflaton requires very large values of the resonance parameter $q, q \gtrsim 10^{8}$. For these large $q$, the maximal size of produced fluctuations is significantly suppressed by the back reaction, but at least within the Hartree approximation they are still not negligible. For the massless inflaton with a $\lambda \phi^{4} / 4$ potential, the Universe expansion completely prevents a resonance production of particles with masses larger than $\sqrt{\lambda} \phi(0)$ for $q$ up to $q=10^{6}$.
\end{abstract}

PACS numbers: 98.80.Cq, 05.70.Fh 
The quantitative theory of reheating after inflation is an important problem in inflationary cosmology [i] because the way the reheating proceeds determines possibility of certain phase transitions and scenarios of baryogenesis. It was recently realized that stimulated processes, due to large occupation numbers of produced bosons, can be most significant during reheating [2 5]. However, despite considerable progress made [2,6,7], still little was known quantitatively about the important case when the coupling of the inflaton field $(\phi)$ to bosonic fields $(X)$ is relatively large (the wide resonance case). It was not clear precisely how wide the resonance should be (for a given mass of $X$ ) to overcome the expansion of the Universe. If the coupling required by this condition turns out to be too large, the resonance will be prevented by non-linear effects, which in this case will set in when $X$ fluctuations are still of the quantum strength. Thus even the existence of a wide resonance in the expanding Universe was not firmly established, let alone its quantitative details. The purpose of the present work was to study the wide resonance case by numerical means.

The main quantity that one wants to know for a given inflationary model is the maximal size of fluctuations of fields produced in the inflaton decay. The size of these fluctuations determines which phase transitions took place after inflation [8,9], whether enough of heavy leptoquarks could be produced to generate the baryon asymmetry [8,9, 7],10], and how strong was the cosmological breakdown of supersymmetry [11, 12].

The reason why fluctuations produced at reheating could be sufficiently large for the above effects to exist is that, as now recognized, reheating in many cases starts with a stage of parametric resonance (called preheating in ref. [2]), during which the bosonic fluctuations grow extremely (exponentially) fast. In the expanding Universe, a parametric resonance is effective only when it is sufficiently wide or when the system is (classically) conformally invariant. Otherwise, the redshift of momenta prevents the resonance from developing [13]. The conformally invariant case is realized when a massless inflaton decays into a massless fluctuation field. Production of a massive field can only take place in the wide resonance regime. In that case, there can occur an interesting and somewhat counterintuitive phenomenon, when quanta of a field that strongly couples to the inflaton can be efficiently produced even if their mass is much larger than the frequency of the inflaton oscillations. The effect can be thought of as a "many $\rightarrow$ few" process, in which many quanta of the inflaton field assemble together to produce a few heavy quanta. If the fields that can be efficiently produced by this mechanism include leptoquarks of a grand unified theory (GUT), the subsequent non-equilibrium decay of these leptoquarks could give rise to a sizable baryon asymmetry [8,9,:7, 10].

In the present work, we wanted, first of all, to find the regions of parameters, for which a wide resonance can overcome the redshift of fields and momenta in the expanding Universe. We considered two models: in the first one, the inflaton potential was $m^{2} \phi^{2} / 2$, in the second, it was $\lambda \phi^{4} / 4$. In both cases, we considered decay of the inflaton into a heavy scalar field $X$ of mass $M_{X}$, due to coupling $\frac{1}{2} g^{2} \phi^{2} X^{2}$. The width of the resonance in these models is determined by parameter $q$ : in Model 1 (a massive inflaton) $q \equiv g^{2} \phi^{2}(0) / 4 m^{2}$, where $\phi(0)$ is the value of the inflaton field when it starts to oscillate:f in Model 2 (the massless inflaton), $q \equiv g^{2} / 4 \lambda$.

${ }^{1}$ For a static universe, in the linear regime with respect to fluctuations, this agrees with the 
In Model 2, we have found no resonance for any $M_{X}>\sqrt{\lambda} \phi(0)$ with $q$ up to $q=10^{6}$. In Model 1 with $M_{X} / m=10$, which may be appropriate for scalar leptoquarks, we have found that the redshift is overcome only for very large $q, q \gtrsim 10^{8}$. Thus, a wide enough resonance can indeed survive in the expanding Universe, though account for the expansion is very important for determining precisely how wide it should be.

Our second objective in this work was to determine the maximal size achieved by $X$ fluctuations in Model 1. In general, both the Universe expansion and the back reaction play a role in determining the maximal size of fluctuations. A fully non-linear calculation along the lines of ref. [5], which would allow us to include consistently all back reaction effects, was technically unfeasible for $q \gg 10^{3}$. So, we took the back reaction into account approximately, using a simple Hartree procedure [2,4].

The Hartree approximation may overestimate the maximal size of fluctuations. The interaction term in the equation of motion for $\phi$ becomes important when $\left\langle X^{2}\right\rangle \sim \phi^{2}(0) / q$ (the brackets denote averaging over space). One might expect that this value signifies the end of the resonance stage. However, in the Hartree approximation, when this value is reached, $\left\langle X^{2}\right\rangle$ merely renormalizes the mass of $\phi$ [2] and continues to grow. We cannot say at present if that further growth is an artifact of the Hartree approximation in this model and will disappear in the fully non-linear picture. In the conformally invariant case (see below), the limited set of data points that we have for the full problem does fit on a $1 / q$ curve, in disagreement with the Hartree approximation. We are not willing to make any inferences for the present case, however, because the time evolution of is rather model dependent.

We neglected any self-coupling of the field $X$. In the Hartree approximation, an estimate shows that a quartic self-coupling $\tilde{\lambda} X^{4} / 4$ can be neglected during the entire resonance stage when $\tilde{\lambda} \lesssim g^{2}$. If the maximal size of fluctuations is in fact smaller than obtained in the Hartree approximation, this self-coupling can be neglected at the resonance stage even for larger $\tilde{\lambda}$.

The resulting $\left\langle X^{2}\right\rangle_{\max }$ as a function of $q$ (the brackets denote averaging over space) is shown in Fig. 11. It is suppressed for large $q$ but is still not negligible. If we measure the strength of the (massive) fluctuations with regard to symmetry restoration [8,9] using the effective "temperature": $T_{\text {eff }}^{2} / 12 \equiv\left\langle X^{2}\right\rangle_{\max }$, then for $q=10^{8}$, the Hartree approximation gives $T_{\text {eff }}$ of order $10^{-3} M_{\mathrm{Pl}}$. This is of order of the GUT scale, so the process could be relevant for symmetry restoration even in Grand Unified models.

One should keep in mind, though, that the size of fluctuations can be further altered (apart from the redshift) by the subsequent chaotic evolution [5], absent in the Hartree approximation. That chaotic evolution is rather fast because it is due to stimulated, i.e. classically enhanced, processes. For example, in applications to GUT baryogenesis, the chaotic evolution will in large part take place before the B-non-conserving decays of $X$ particles. Therefore, some quantitative understanding of it is required before reliable estimates of the baryon asymmetry can be made; this will be a subject of a forthcoming paper.

Below we describe our models and calculations in more detail.

definition of the parameter $q$ of the canonical Mathieu equation [14]. 
Model 1: Massive inflaton. In conformal variables, and after a convenient rescaling, the action for the matter fields is

$$
\begin{gathered}
S_{m}=\frac{\phi^{2}(0)}{m^{2}} \int d^{3} \xi d \tau\left[\frac{1}{2}\left(\dot{\varphi}-\frac{\dot{a}}{a} \varphi\right)^{2}-\frac{(\nabla \varphi)^{2}}{2}+\frac{1}{2}\left(\dot{\chi}-\frac{\dot{a}}{a} \chi\right)^{2}-\frac{(\nabla \chi)^{2}}{2}-\frac{1}{2} \frac{a^{2}(\tau)}{a^{2}(0)} \varphi^{2}\right. \\
\left.-\frac{1}{2} \frac{M_{X}^{2}}{m^{2}} \frac{a^{2}(\tau)}{a^{2}(0)} \chi^{2}-2 q \varphi^{2} \chi^{2}\right],
\end{gathered}
$$

where $\tau=m a(0) \eta, \eta$ is the conformal time; $\xi=m a(0) x ; \varphi=\phi a(\tau) / \phi(0) a(0), \chi=$ $X a(\tau) / \phi(0) a(0) ; a(\tau)$ is the scale factor of the Universe.

We take $\tau=0$ at the moment of the first extremum of $\phi(\tau)$. Solving the equation of motion for the zero-momentum mode of the inflaton coupled to gravity, together with the Einstein equation for the scale factor, we have found $\phi(0)=0.28 M_{\mathrm{Pl}}$. At later times, we continued to determine the evolution of the scale factor self-consistently, including the influence of produced fluctuations in the Einstein equations. After $\tau=0$, a matter dominated stage quickly sets in, for which one can use

$$
\frac{a(\tau)}{a(0)}=\left(\sqrt{\frac{\pi}{3}} \frac{\phi(0)}{M_{\mathrm{Pl}}} \tau+1\right)^{2} \approx(0.29 \tau+1)^{2} .
$$

However, as our self-consistent solution confirms, for some values of the parameters the expansion later crosses over into a radiation dominated stage. That happens when the interaction potential $\frac{1}{2} g^{2} \phi^{2} X^{2}$ overtakes the mass term of $\phi$.

In the Hartree approximation, the zero-momentum mode is the only non-vanishing mode of the field $\varphi$; we keep for it the notation $\varphi$. Its equation of motion is

$$
\ddot{\varphi}-\frac{\ddot{a}}{a} \varphi+\frac{a^{2}(\tau)}{a^{2}(0)} \varphi+4 q\left\langle\chi^{2}\right\rangle \varphi=0,
$$

where the brackets denote an average over space. This should be solved with the initial conditions $\varphi(0)=1, \dot{\varphi}(0)=0$. The equation of motion for the Fourier components of the field $\chi$ is

$$
\ddot{\chi}_{\mathbf{k}}+\omega_{k}^{2}(\tau) \chi_{\mathbf{k}}=0
$$

where

$$
\omega_{k}^{2}(\tau)=k^{2}-\frac{\ddot{a}}{a}+\frac{M_{X}^{2}}{m^{2}} \frac{a^{2}(\tau)}{a^{2}(0)}+4 q \varphi^{2}(\tau)
$$

We will sometimes use the notation

$$
m_{\chi}^{2} \equiv \frac{M_{X}^{2}}{m^{2}}
$$

The Fourier components are normalized in such a way that the variance of the fluctuation field is 


$$
\left\langle\chi^{2}\right\rangle=\frac{1}{(2 \pi)^{3}} \int d^{3} k\left|\chi_{\mathbf{k}}\right|^{2}
$$

Because fluctuations are produced mostly with relatively small values of (comoving) momenta $k$, the inevitable presence of a momentum cutoff in our calculation, somewhere at larger momenta, will automatically result in a properly renormalized $\left\langle\chi^{2}\right\rangle$. If we wanted to embed our calculation into a fully quantum-field-theoretical picture, we would identify the parameters we use $\left(m, g^{2}\right.$ etc.) with the running parameters of that picture, taken at a normalization point of the order of our momentum cutoff; moderate changes in the normalization point will only cause corrections of relative order $g^{2} / 4 \pi=q m^{2} / 4 \pi \phi^{2}(0)$.

The initial conditions for $\chi_{\mathbf{k}}$ are randomly distributed with the probability density

$$
\mathcal{P}_{k}\left[\chi_{\mathbf{k}}\right] \propto \exp \left[-\frac{2 \phi^{2}(0)}{m^{2}} \omega_{k}(0)\left|\chi_{\mathbf{k}}(0)\right|^{2}\right] .
$$

According to eq. (1), $m^{2} / \phi^{2}(0)$ is the parameter that regulates the strength of quantum fluctuations compared to classical ones in this model. The initial "velocities" are locked to their corresponding "coordinates"

$$
\dot{\chi}_{\mathbf{k}}(0)=\left[-i \omega_{k}(0)+h(0)\right] \chi_{\mathbf{k}}
$$

where $h=\dot{a} / a$. For a derivation of these initial conditions, see ref. [5]. The semiclassical description is reliable as long as $g^{2} / 4 \pi=q m^{2} / 4 \pi \phi^{2}(0) \ll 1$.

The results of numerical calculations for the realistic case $m=10^{-6} M_{\mathrm{Pl}}$ are presented in Figs. 1 1 .

Three factors can potentially suppress a parametric resonance in the expanding Universe, each represented by the corresponding term in the equations of motion. In our conformal variables, they are: the time dependent mass term $\propto M_{X}^{2}$ in eq. (5), which redshifts the modes away from the resonance; the time dependent mass term of the field $\varphi$ in eq. (3), which makes the oscillations of the background field aperiodic; and the back reaction term $\propto q$ in eq. (3). A very useful quantity to introduce is the effective $q$,

$$
q_{\mathrm{eff}}(\tau) \equiv \frac{g^{2} \bar{\phi}^{2}(\tau)}{4 m_{\mathrm{eff}}^{2}(\tau)},
$$

where $\bar{\phi}$ is the (decreasing) amplitude of the physical field $\phi$, and $m_{\text {eff }}$ is that field's effective mass including the Hartree correction. In a slowly expanding Universe (the adiabatic limit), $q_{\text {eff }}(\tau)$ would indeed be the correct resonance parameter at time $\tau$.

To understand the effects of the redshift and the back reaction, it is convenient to see first what happens when the back reaction is neglected. This is indeed the correct limit for sufficiently small $q$ (a more precise criterion depends on $m_{\chi}$ ). Then, $m_{\mathrm{eff}}=m$ and

$$
q_{\mathrm{eff}}(\tau)=q \frac{\bar{\phi}^{2}(\tau)}{\bar{\phi}^{2}(0)}
$$

For a parametric resonance to be effective at time $\tau, q_{\text {eff }}(\tau)$ should not be much smaller than unity. Because a resonance will need some time $\tau_{0}$ to start, the condition for it to begin at 
all, $q_{\text {eff }}\left(\tau_{0}\right) \gtrsim 1$, already requires large values of $q=q_{\text {eff }}(0)$. By our definition, a resonance starts when $\left\langle X^{2}\right\rangle$ rises above quantum fluctuations. E.g. in Fig. 2, $\tau_{0} \approx 6$; some $k$-modes can start to grow earlier, however.

Consider first the case of massless $X, M_{X}=0$. We have found that even then the resonance starts only for $q \gtrsim 10^{3}$, see Fig. 1. With $q \gtrsim 10^{3}$, the parametric resonance starts at some point but terminates when $q_{\text {eff }}$ drops below unity. Hence, in the expanding Universe, the resonance stops and the maximal size of produced fluctuations is limited in this model] even without back reaction. We have calculated the maximum value of $\left\langle X^{2}\right\rangle$ that would be achieved without back reaction for a wide range of values of $q$. It is plotted in Fig. I by the dotted curve and for massless $X$ has the label $m_{\chi}=0$. This curve can be approximated by $\left\langle X^{2}\right\rangle_{\max } \propto q^{12}$, which is plotted in Fig. 11 by a solid straight line.

Next, consider the case $M_{X} \neq 0$. The rapidly growing mass term of $\chi$ is a resonance suppressing factor. In the case of the $\lambda \phi^{4}$ model, we will see that it works against the resonance extremely effectively. In the present model, however, the frequency of oscillations of the field $\varphi$ is growing at the same rate as the effective mass of $\chi$, compare eqs. (3) and (5). In a sense, massive $\chi$ modes can remain tuned to the resonance, in some analogy with the conformal case. Still, for a non-zero $M_{X}$, the resonance terminates before $q_{\text {eff }}$ drops below unity. This is why the curves with $m_{\chi} \neq 0$ are shifted in Fig. 1 to the right with respect to the $m_{\chi}=0$ case.

In the absence of the Universe expansion and back reaction, the linearized equation for fluctuations, eq. (4), would be a Mathieu equation. In that case, a necessary condition for the $X$ particle production to fall into the strongest resonance band would be, for $m_{\chi} \gtrsim 1$ : $m_{\chi} \lesssim q^{1 / 4}$, or equivalently $q \gtrsim\left(M_{X} / m\right)^{4}$. As one can see from Fig. 1, this estimate is four orders of magnitude off in the expanding Universe: e.g. with $q \sim 10^{4}$ the resonance barely develops even for $m_{\chi}=1$. To take the Universe expansion into account, we can replace $q$ in the above estimate with $q_{\text {eff }}$ at some time before the end of the resonance stage, $q_{\text {eff }}\left(\tau_{1}\right)$. As evident from eq. (11), finding $q_{\mathrm{eff}}\left(\tau_{1}\right)$ is equivalent to finding $\bar{\phi}\left(\tau_{1}\right)$. The quantities $\tau_{1}$ and $\bar{\phi}\left(\tau_{1}\right)$ in general depend on $q$. Although some analytical estimates of $\bar{\phi}\left(\tau_{1}\right)$ can be made [8], its reliable determination requires our numerical results. We have found that for the range of $q$ we consider in Fig. 1, one can use generically $\bar{\phi}^{2}\left(\tau_{1}\right) \sim 10^{-4} \phi^{2}(0)$. Using $q_{\text {eff }}$ instead of $q$ repairs the estimate: to efficiently produce particles of mass $M_{X}$, one needs $q_{\text {eff }}\left(\tau_{1}\right) \gtrsim\left(M_{X} / m\right)^{4}$, or $q \gtrsim 10^{4}\left(M_{X} / m\right)^{4}$, in agreement with Fig. 1. For $M_{X} / m=10$, we get $q \gtrsim 10^{8}$, which for $m=10^{13} \mathrm{GeV}$ translates into $g^{2} \gtrsim 4 . \times 10^{-3}$. Considering the complexity of the phenomena involved, the estimate $g^{2} \gtrsim 10^{-4}$ made for this case in ref. [10] is in a reasonable agreement with our result.

The term "wide" parametric resonance is to some extent misleading. The resonance band itself is narrow, $\Delta k \ll q_{\mathrm{eff}}^{1 / 4}$. However, the resonance band can be anywhere in the interval $0<k_{\text {res }} \lesssim q_{\text {eff }}^{1 / 4}$, and indeed moves through this interval back and forth when $q_{\text {eff }}$ slightly changes. Now, since $q_{\text {eff }}$ is time dependent in expanding Universe, all $k$ from this

${ }^{2}$ As opposed to the conformally invariant case [2, 15,5]. 
range become resonant in turn, $]^{3}$ and it is for that reason that one may consider $q_{\mathrm{eff}}^{1 / 4}$ as a kind of effective width of the resonance. As a result of the motion of the resonance band, all $k$ up to $q^{1 / 4}$ become filled up in the power spectrum, although to a varying extent.

In Fig. 1, notice also the sensitivity of the maximal size of fluctuations with respect to small changes in $q$. Small changes in $q$ can be simulated by small numerical uncertainties, such as that of the coefficient in the relation $\phi(0)=0.28 M_{\mathrm{Pl}}$. It anyway makes sense to average out the rapid changes in Fig. 1 and concentrate on the general pattern. As a general trend, we observe a broad maximum of $\left\langle X^{2}\right\rangle_{\max }$ as a function of $q$, at fixed $M_{X}$. It occurs in the region of $q$ where the redshift and the back reaction play comparable roles in terminating the resonance.

Now consider the limit when the maximum value attained by fluctuations is determined by the back reaction. This is the case of asymptotically large $q$. The general trend in $\left\langle X^{2}\right\rangle_{\max }$ in this region of $q$ is given by

$$
\left\langle X^{2}\right\rangle_{\max } \sim \frac{3 \times 10^{-3}}{\sqrt{q}} M_{\mathrm{Pl}}^{2}
$$

which is plotted in Fig. 1 by the dashed straight line. In this region, we can also estimate $\left\langle X^{2}\right\rangle_{\max }$ in the Hartree approximation analytically, and the estimate is in rather good agreement with eq. (12). It proceeds as follows. We first notice that because the decay of $\phi$ into $X$ is a stimulated process, it mostly takes place in a short period close to the end of the resonance, when $X$ fluctuations are already large. Then, we should distinguish between the amplitude of the inflaton oscillations just before the decay, $\bar{\phi}\left(\tau_{1}\right)$, which is determined mostly by the redshift, and its amplitude right after the decay, $\bar{\phi}\left(\tau_{2}\right)$. For a wide resonance, $\bar{\phi}\left(\tau_{2}\right)$ can be smaller than $\bar{\phi}\left(\tau_{1}\right)$ by orders of magnitude. Such a drop is clearly seen in Fig. 3 (even though for $m_{\chi}=10$ the value $q=10^{8}$ is not an asymptotically large $q$ ). Because $\tau_{2}-\tau_{1}$ is such a short time, the Universe expansion during it can be neglected, and from energy conservation we get

$$
g^{2} \bar{\phi}^{2}\left(\tau_{2}\right)\left\langle X^{2}\right\rangle \sim m^{2} \bar{\phi}^{2}\left(\tau_{1}\right)
$$

The second ingredient of our estimate is the observation [2] that the presence of $\left\langle X^{2}\right\rangle$ changes the effective mass of the inflaton; the effective mass is given by $m_{\text {eff }}^{2}=m^{2}+g^{2} \overline{\left\langle X^{2}\right\rangle}$, where $\overline{\left\langle X^{2}\right\rangle}$ is a slowly changing part of $\left\langle X^{2}\right\rangle$. When $\left\langle X^{2}\right\rangle$ becomes sufficiently large, $m_{\text {eff }}^{2} \approx$ $g^{2} \overline{\left\langle X^{2}\right\rangle} \sim g^{2}\left\langle X^{2}\right\rangle$, and the effective resonance parameter becomes $q_{\mathrm{eff}}=g^{2} \bar{\phi}^{2} / 4 m_{\mathrm{eff}}^{2} \sim$ $\bar{\phi}^{2} /\left\langle X^{2}\right\rangle$. The resonance stops when $q_{\mathrm{eff}}\left(\tau_{2}\right) \sim 1$; using eq. (13), this givesf

$$
\left\langle X^{2}\right\rangle_{\max } \sim \frac{m \bar{\phi}\left(\tau_{1}\right)}{g}
$$

\footnotetext{
${ }^{3}$ It is perhaps not obvious a priori that this motion of the resonance band does not kill the resonance altogether.

${ }^{4}$ Our usual condition $q_{\text {eff }} \gtrsim\left(M_{X} / m_{\text {eff }}\right)^{4}$ is satisfied for $q_{\text {eff }} \sim 1$ at sufficiently large $q$, due to the large $m_{\mathrm{eff}}$. Notice also that because a large $m_{\mathrm{eff}}$ lowers the $M_{X} / m_{\mathrm{eff}}$ ratio, the Hartree correction to
} 
The value of $\bar{\phi}\left(\tau_{1}\right)$ is weakly depends on $q$. As before, we use the generic estimate $\bar{\phi}\left(\tau_{1}\right) \sim 10^{-2} \phi(0)$ for all $q$ considered. We then obtain $\left\langle X^{2}\right\rangle_{\max } \sim 10^{-3} M_{\mathrm{Pl}}^{2} / \sqrt{q}$, in reasonable agreement with the numerical result (12). A $1 / \sqrt{q}$ (up to logarithms) estimate for $\left\langle X^{2}\right\rangle_{\text {max }}$ appears in refs. [2,8, 11], although the way the estimate is obtained and the numerical coefficient are somewhat different from ours. We stress again that we do not know at present if the $1 / \sqrt{q}$ behavior is an artifact of the Hartree approximation in this model. See also the discussion of the conformally invariant case below.

Note that at asymptotically large $q$, the interaction term $\frac{1}{2} g^{2} X^{2} \phi^{2}$ dominates over the mass term of $X$ during the entire resonance stage. So, at the resonance stage, the fluctuations of $X$ cannot really be described as particles, since they are strongly coupled to the oscillating background.

Model 2: Massless inflaton. In rescaled variables, the action for the matter fields in this model is

$$
\begin{gathered}
S_{m}=\frac{1}{\lambda} \int d^{3} \xi d \tau\left[\frac{1}{2}\left(\dot{\varphi}-\frac{\dot{a}}{a} \varphi\right)^{2}-\frac{(\nabla \varphi)^{2}}{2}+\frac{1}{2}\left(\dot{\chi}-\frac{\dot{a}}{a} \chi\right)^{2}-\frac{(\nabla \chi)^{2}}{2}-\frac{1}{4} \varphi^{4}\right. \\
\left.-\frac{1}{2} \frac{M_{X}^{2}}{\lambda \phi^{2}(0)} \frac{a^{2}(\tau)}{a^{2}(0)} \chi^{2}-2 q \varphi^{2} \chi^{2}\right]
\end{gathered}
$$

where $\varphi$ and $\chi$ are defined as before but $\tau=\sqrt{\lambda} \phi(0) a(0) \eta, \xi=\sqrt{\lambda} \phi(0) a(0) x$.

We again take $\tau=0$ to be the first extremum of $\phi(\tau)$. In this case we find $\phi(0)=0.35 M_{\mathrm{Pl}}$. There is no resonance for a massive enough $X$ (see below). In that case, after the inflaton oscillations start, the Universe quickly becomes radiation dominated and we can use

$$
\frac{a(\tau)}{a(0)}=\sqrt{\frac{2 \pi}{3}} \frac{\phi(0)}{M_{\mathrm{Pl}}} \tau+1 \approx 0.51 \tau+1
$$

All other formulas are obtained from the corresponding ones for Model 1 by replacing $m^{2}$ with $\lambda \phi^{2}(0)$. Accordingly, we now use $m_{\chi}=M_{X} / \sqrt{\lambda} \phi(0)$.

For a massless field $\chi$ the problem becomes conformally equivalent to the one in the Minkowski space-time: the expansion of the Universe decouples in the conformal coordinates. The parametric resonance is always effective. We have done a complete non-linear analysis of this problem in the range $1 \leq q \leq 1000$, using the approach developed in ref. [5]. At $q \gg 1$, the evolution of the system is fast and we were able to trace the semiclassical thermalization [5] up to a slowly evolving state with a developed Kolmogorov turbulence. These results will be described in more detail elsewhere; for previous discussions of kinetic phenomena after preheating, see refs. [5, 16]. For the limited set of values of $q$ for which we now have the data, $\left\langle X^{2}\right\rangle$ at the end of the resonance stage in the full problem fits a $1 / q$ curve. If

the mass of $\phi$ actually helps the resonance for a while, as we have indeed observed in our numerical results. 
confirmed by more extensive calculations, such behavior would be in disagreement with the approximately $1 / \sqrt{q}$ dependence obtained in the Hartree approximation.

With a massive $\chi$ field we have not found any parametric resonance for $m_{\chi}>1$ and $q$ up to $q=10^{6}$. Analytically, the condition for the resonance to exist at time $\tau$ (neglecting back reaction) is $q^{1 / 4} \gtrsim m_{\chi} a(\tau) / a(0)$. In the conformally invariant case $\left(m_{\chi}=0\right)$, the fluctuations reach appreciable strength at $\tau \sim 100$. Using that value in the estimate for $m_{\chi} \neq 0$, we would expect that for $m_{\chi}=1$, the resonance exists when $q \gtrsim 10^{7}$. We indeed observed a slow (physically insignificant) resonance at $m_{\chi}=1$ and $q=10^{8}$.

In conclusion, we have studied the decay of massive and massless inflatons into massive excitations in the expanding Universe. We have found that a wide enough resonance can survive in the expanding Universe, though the expansion is very important for determining precisely how wide it should be. For a massive inflaton, the effective production of particles with mass ten times that of the inflaton requires very large values of the resonance parameter $q, q \gtrsim 10^{8}$. For these large $q$, the maximal size of produced fluctuations is significantly suppressed by the back reaction, but at least within the Hartree approximation they are still not negligible. We do not know at present if this conclusion will persist in the fully non-linear picture; the full account for rescattering is thus an important problem for the future. For the massless inflaton with a $\lambda \phi^{4} / 4$ potential, the Universe expansion completely prevents a resonance production of particles with masses larger than $\sqrt{\lambda} \phi(0)$ for $q$ up to $q=10^{6}$.

We thank G. Dvali, L. Kofman, E. Kolb, A. Linde, A. Riotto, V. Rubakov, M. Shaposhnikov, and N. Tetradis for discussions and comments. S.K. thanks CERN Theory Division, where some of the discussions took place, for hospitality. The work of S.K. was supported in part by the U.S. Department of Energy under Grant DE-FG02-91ER40681 (Task B), by the National Science Foundation under Grant PHY 95-01458, and by the Alfred P. Sloan Foundation. The work of I.T. was supported by DOE Grant DE-AC02-76ER01545 at Ohio State. 


\section{REFERENCES}

[1] A. D. Linde, Particle Physics and Inflationary Cosmology (Harwood, Chur, Switzerland, 1990); E. W. Kolb and M. S. Turner, The Early Universe (Addison-Wesley, Redwood City, California, 1990).

[2] L. Kofman, A. Linde, and A. A. Starobinsky, Phys. Rev. Lett. 73, 3195 (1994).

[3] J. H. Traschen and R. H. Brandenberger, Phys. Rev. D 42, 2491 (1990); Y. Shtanov, J. Traschen, and R. Brandenberger, Phys. Rev. D 51, 5438 (1995).

[4] D. Boyanovsky, H. J. de Vega, R. Holman, D.-S. Lee, and A. Singh, Phys. Rev. D 51, 4419 (1995); D. Boyanovsky, M. D’Attanasio, H. J. de Vega, R. Holman, and D.-S. Lee, Phys. Rev. D 52, 6805 (1995).

[5] S. Khlebnikov and I. Tkachev, Phys. Rev. Lett. 77, 219 (1996).

[6] M. Yoshimura, Prog. Theor. Phys. 94, 873 (1995); H. Fujisaki, K. Kumekawa, M. Yamaguchi, and M. Yoshimura, Phys. Rev. D 53, 6805 (1996); hep-ph/9511381.

[7] M. Yoshimura, hep-ph/9605246.

[8] L. Kofman, A. Linde, and A. A. Starobinsky, Phys. Rev. Lett. 76, 1011 (1996).

[9] I. I. Tkachev, Phys. Lett. B376, 35 (1996).

[10] E. W. Kolb, A. D. Linde, and A. Riotto, hep-ph/9606260.

[11] G. W. Anderson, A. Linde, and A. Riotto, hep-ph/9606416.

[12] G. Dvali and A. Riotto, hep-ph/9606431.

[13] J. Preskill, M. B. Wise, and F. Wilczek, Phys. Lett. 120B, 127 (1983); L. F. Abbott and P. Sikivie, Phys. Lett. 120B, 133 (1983); I. I. Tkachev, Sov. Astron. Lett. 12, 305 (1986); A. D. Dolgov and K. Freese, Phys. Rev. D 51, 2693 (1995).

[14] See e.g. M. Abramowitz and I. Stegun, Handbook of Mathematical Functions, Dover, New York, 1972.

[15] D. I. Kaiser, Phys. Rev. D 53, 1776 (1996).

[16] D. T. Son, hep-ph/9604340. 


\section{FIGURES}

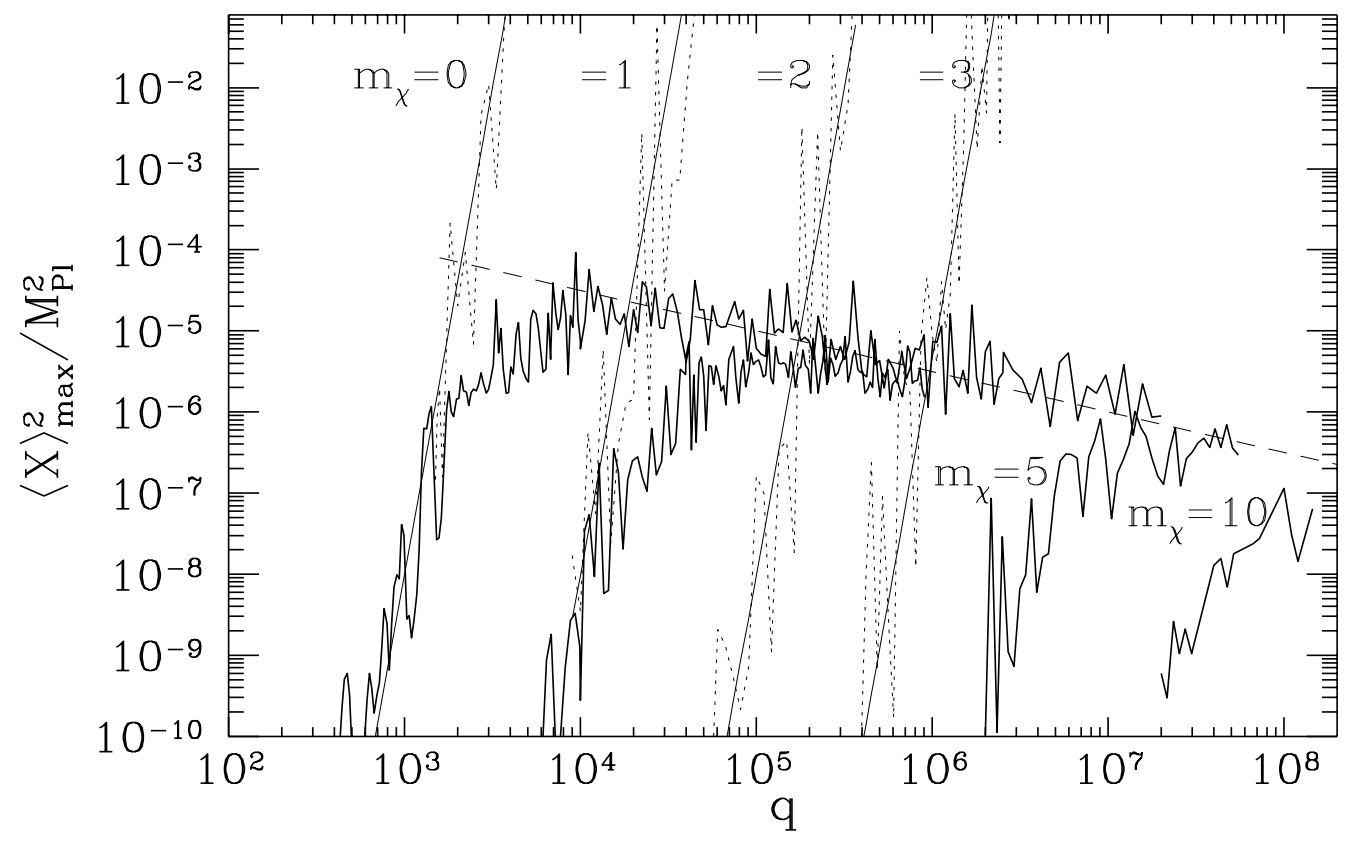

FIG. 1. Maximal value of the variance of fluctuations in Model 1 as a function of the resonance parameter. Dotted lines correspond to no back reaction and are fitted by a power law. Solid curves are obtained in the Hartree approximation. The dashed straight line is a power law fit to the Hartree data.

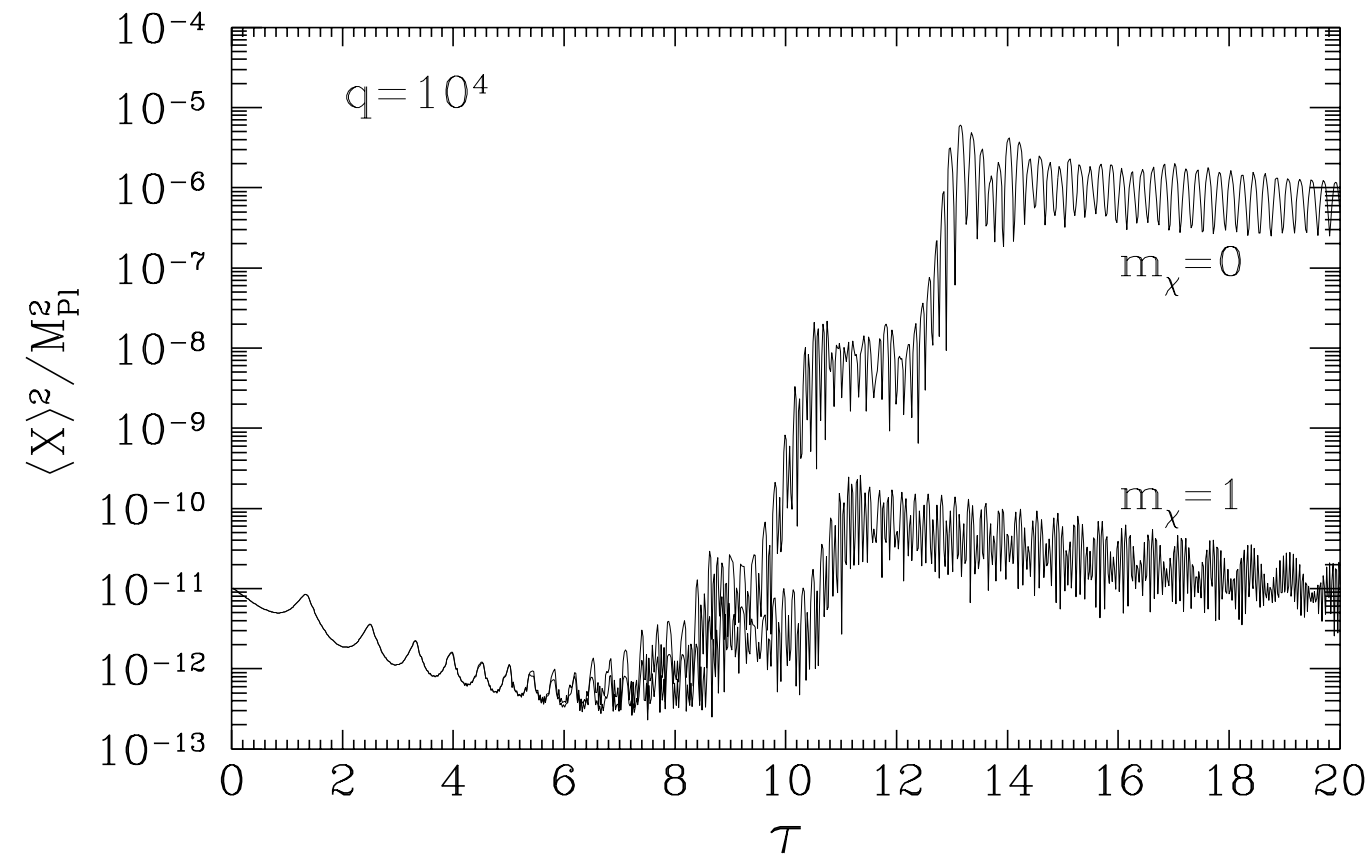

FIG. 2. Fluctuation variance in Model 1, as a function of time, for two values of the mass of the fluctuating field; back reaction of fluctuations on the inflaton field was included in the Hartree approximation. 


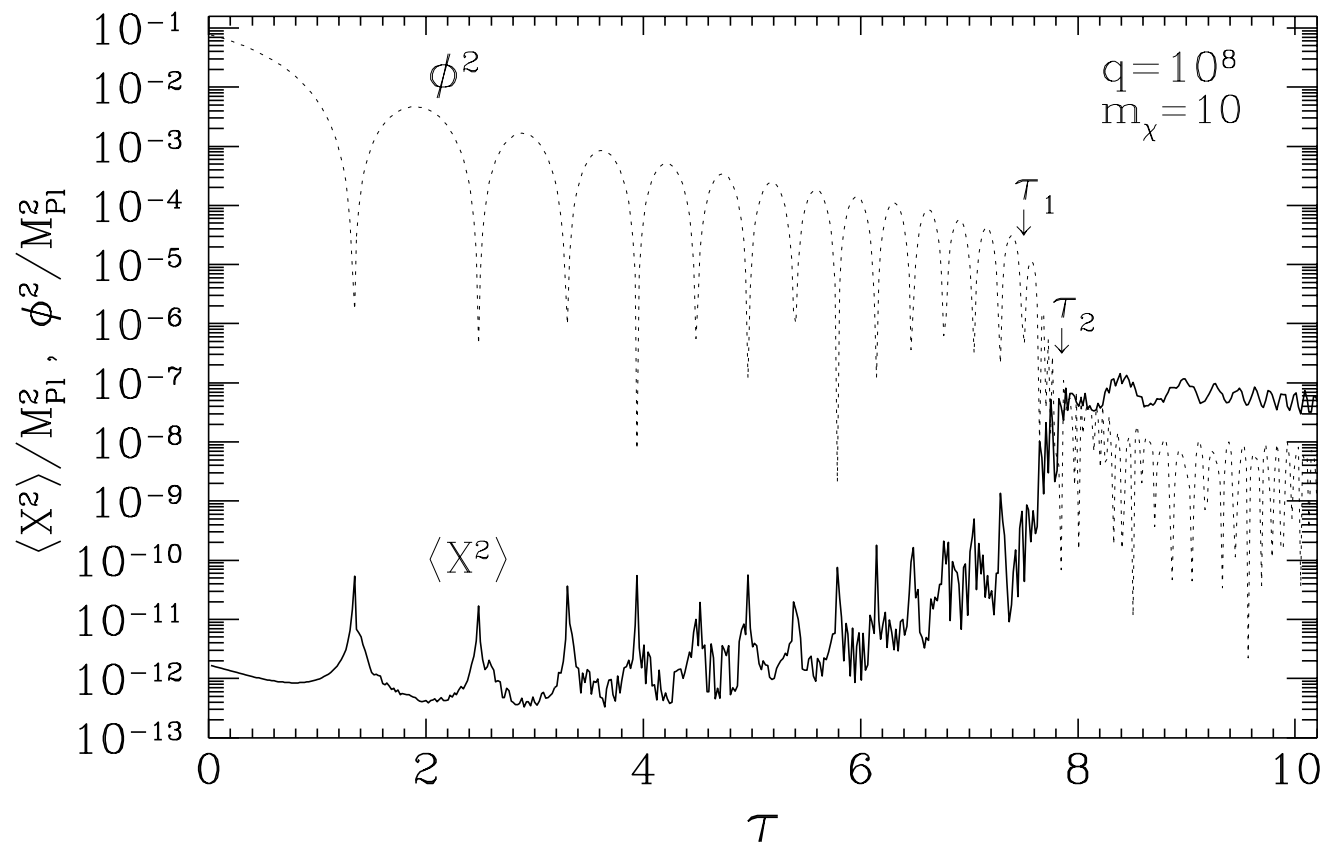

FIG. 3. Time evolution of the homogeneous inflaton field and the fluctuation variance in Model 1 in the Hartree approximation, for given values of the parameters. 